\title{
Análisis de macroinvertebrados bentónicos e índices biológicos para evaluar la calidad del agua del Río Sardinas, Chocó Andino Ecuatoriano
}

\section{(Analysis of benthic macroinvertebrates and biotic indices to evaluate water quality in Sardina's River, Ecuadorian Chocó Andino)}

\author{
Verónica Machado ${ }^{1}$, Roberto Granda ${ }^{1}$, Alexandra Endara ${ }^{1}$
}

\begin{abstract}
Resumen:
Se realizó un análisis de la entomofauna acuática del Río Sardinas, en las épocas lluviosa y seca de 2018, usando macroinvertebrados como bioindicadores ambientales, los mismos que fueron recolectados con red Surber, red de patada y colecta manual, en cinco puntos a lo largo del río. En laboratorio fueron clasificados e identificados a niveles de clase, orden y familia. Se analizó su riqueza (S) y abundancia (N), así como la calidad ambiental aplicando los índices BMWP/Col, EPT y un Análisis de Componentes Principales PCA. Se recolectaron un total de 526 individuos pertenecientes a 5 clases, 13 órdenes y 38 familias; 31 en época lluviosa y 30 en época seca. La familia más abundante en lluvias fue Leptophlebiidae, 34 especímenes (12,8\%); en la estación seca, la más representativa fue Chironomidae con 45 individuos (17,2\%). En la estación lluviosa el punto 2 tuvo mayor riqueza S15. En la estación seca P1, S18. Los puntos P4 - P5 presentaron mediana calidad (BMWP/Col=69-94) y un EPT de 56 en lluvias y 38 en estación seca. En este estudio se determinó el estado ecológico del Río Sardinas con base a su abundancia, riqueza, índices BMWP/Col, EPT y la influencia de los parámetros físico-químicos mediante un PCA.
\end{abstract}

Palabras clave: entomofauna acuática; calidad ambiental; bosque piemontano

\begin{abstract}
:
An aquatic macroinvertebrates community analysis was made at Sardinas River, in the rainy and wet seasons of April and July, 2018, using macroinvertebrates as environmental bioindicators, these were collected with Surber net, kick net and hand collection, in five points along the river. In laboratory they were classified and identified as class, order and family. They were studied by analysis of richness (S) and abundance (N). The environmental quality was determinate applying the BMWP/Col, EPT indices and Principal component analysis. A total of 526 individuals belonging to five class, 11 orders and 36 families were collected, 31 families in rainy season and 30 in dry season. The most abundant family in rainy season was Leptophlebiidae, 34 specimens, $12,8 \%$. In dry season, the most representative was Chironomidae with 45 individuals, $17.2 \%$. In rainy season the points 2 had greater richness $\mathrm{S} 15$ and in the dry season P1, S18, P3 - P5 presented medium quality water $(\mathrm{BMWP} / \mathrm{Col}=69-94)$ and an EPT of 56 wet season and 38 dry season. This study determined the ecological status of Sardinas river through abundance, richness, BMWP/Col, EPT index and Physico-chemical parameters using a PCA.
\end{abstract}

Keywords: aquatic entomofauna; environmental quality; Andean montane forest.

\footnotetext{
1 Universidad UTE, Quito, Ecuador ( \{mzvs100734, roberto.granda, maria.endara\} @ute.edu.ec).
} 


\section{Introducción}

El acelerado crecimiento de la población humana, la industrialización y la urbanización son causas directamente asociadas a la contaminación del agua (Courtemanch et al., 1989); en la Zona Pacto, Chocó Andino, los cambios de uso de suelo, el crecimiento poblacional, el desarrollo de actividades industriales (minería aurífera) y agrícolas (uso de agroquímicos), han originado el deterioro de los sistemas hídricos de la misma (Jiménez et. al., 2011).

El Río Sardinas está sujeto a presiones humanas que afectan a los recursos hídricos de la región, de esta manera los pobladores reconocen que estos impactos negativos, han causado la disminución de calidad y cantidad del agua (PDOT Pacto, 2015 - 2019). Como consecuencia existe un creciente interés por conocer y proteger los ecosistemas acuáticos mediante análisis biológicos (Figueroa et. al., 2003), para reconocer el grado de alteración del cuerpo de agua y mediante su análisis brindar alternativas de control, rehabilitación y recuperación hídrica.

La valoración biótica es una evaluación que analiza los cambios en las condiciones del cuerpo de agua, estudiando la sensibilidad de la biota a los contaminantes presentes. Estos cambios han motivado en las últimas décadas el desarrollo de índices bióticos para valorar el efecto de las intervenciones humanas sobre dichos ecosistemas (González et. al., 2013).

Con estos antecedentes se pudo determinar la necesidad de realizar un análisis de la calidad del agua del Río Sardinas, utilizando a los macroinvertebrados bentónicos como organismos para valorar la integridad del estado ecológico de las masas de agua, ya que la estructura y composición de sus comunidades, permiten identificar modificaciones físicas y químicas del ecosistema acuático (Tomanova y Tedesco, 2007; Instituto Nacional de Ecología y Cambio Climático [INECC] y El Colegio de la Frontera Sur [Ecosur], 2014).

Los índices biológicos se basan en asociar la taxa de los macroinvertebrados con su nivel de tolerancia a la contaminación (Figueroa et. al. 2003). Se les asigna un número cuyo rango varía según la metodología que se emplee. El índice BMWP, el más empleado en Latinoamérica, junto con el índice EPT (Ephemeroptera, Plecoptera, Trichoptera) son utilizados para el seguimiento de la salud ambiental de los ecosistemas dulceacuícolas (Gamboa et. al., 2008).

Los índices de diversidad se basan en las variaciones de las comunidades bióticas y señalan que a una mayor biodiversidad, la calidad del agua es mejor (Carrera, 2011). Estos índices son empleados de manera conjunta, considerando la riqueza taxonómica (índices cualitativos) o la abundancia relativa (índices cuantitativos), para determinar el estado de agua (Springer, 2010). Los análisis de los parámetros de riqueza y abundancia sirven para evaluar el estado ecológico del área de estudio (Dousdebés, 2016).

El objetivo de esta investigación es determinar el estado ecológico y la calidad de agua del Río Sardinas en épocas seca y lluviosa, utilizando a los macroinvertebrados presentes como bioindicadores de calidad ambiental, mediante la evaluación de su biodiversidad y su relación con los parámetros físico - químicos.

\section{Metodología}

\section{1. Área de estudio}

El río Sardinas se ubica en Ecuador, en la provincia de Pichincha, cantón Quito, parroquia de Pacto. Nace en la loma Esperanza, de vertientes naturales, su extensión aproximada es de $40 \mathrm{~km}$, desemboca en el río Guaycuyacu que posteriormente se conecta y desemboca en el río Guayllabamba (Villalva, 2018). Presenta un bioclima tropical megatérmico muy húmedo, se identifican dos épocas climáticas: 1) época lluviosa, presente de diciembre a mayo, y 2) época seca, presente de junio a noviembre 
(Ministerio de Agricultura y Ganadería - Instituto Espacial Ecuatoriano [MAGAP - IEE], 2013). La pluviosidad varía entre los $2000 \mathrm{~mm}$ y $4000 \mathrm{~mm}$ de precipitación anual y la temperatura oscila entre 12 y $20{ }^{\circ} \mathrm{C}$ (Herrera, 2018). Los puntos de muestreo se encuentran en la formación vegetal bosque siempreverde piemontano de Cordillera Occidental de Los Andes BsBn04 (MAE, 2013).

El área estudiada del Río Sardinas se encuentra entre 893 y $1050 \mathrm{msnm}$, en donde se hicieron dos muestreos, uno en época de lluvias (20 de abril del 2018) y otro, en la época seca (22 de julio de 2018). Se establecieron cinco puntos de colecta, separados entre sí por $200 \mathrm{~m}$, a lo largo del gradiente ecológico del río, desde bosque sin intervención humana hasta zonas de cultivos y pastos (Figura 1, Tabla 1). Las coordenadas y la altitud se registraron con un GPS Garmin, Oregon 550.
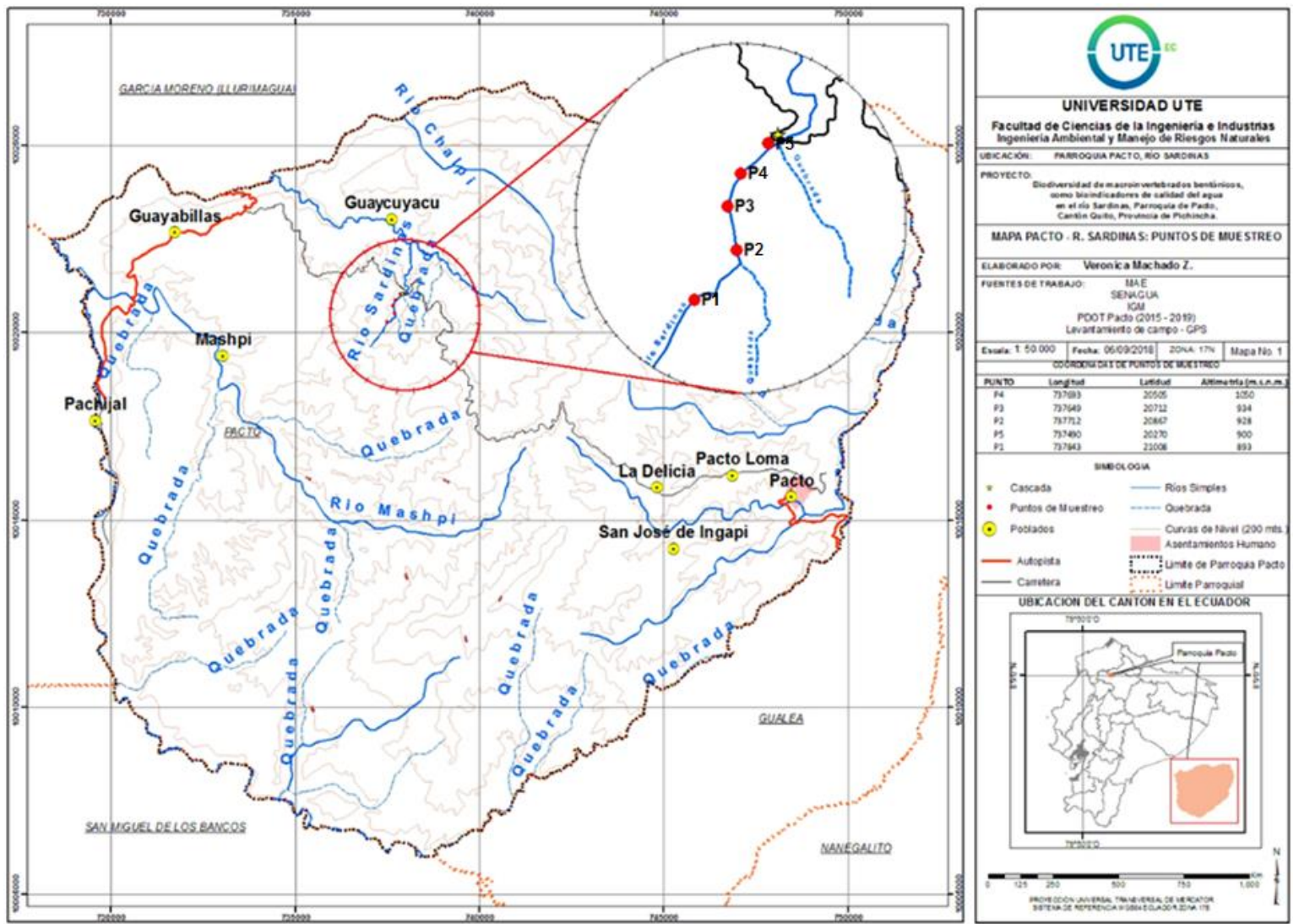

Figura 1. Ubicación de los puntos de muestreo en el río Sardinas, parroquia Pacto.

El estudio se realizó en dos fases: la primera, de campo, donde se procedió al establecimiento de los puntos de muestreo, captura de especímenes, toma de muestras del agua y mediciones de los parámetros físico - químicos. La segunda fase se realizó en el laboratorio, y consistió en la identificación y análisis biológico de los individuos colectados mediante índices y análisis físico-químico de las muestras de agua colectadas.

\subsection{Fase de campo:}

Para la recolección de los especímenes se utilizaron tres técnicas, con la finalidad de cubrir todos los microhábitats posibles y especies asociadas (Abarca, 2007). 
Tabla 1. Coordenadas geográficas y características de los puntos de muestreo de macroinvertebrados acuáticos en el Río Sardinas.

\begin{tabular}{|c|c|c|c|c|c|c|c|c|}
\hline \multirow{2}{*}{$\begin{array}{l}\text { Puntos } \\
\text { de } \\
\text { muestreo }\end{array}$} & \multirow[b]{2}{*}{ Características } & \multicolumn{2}{|c|}{$\begin{array}{l}\text { Coordenadas } \\
\text { UTM }\end{array}$} & \multirow{2}{*}{$\begin{array}{l}\text { Altitud } \\
\text { (msnm) }\end{array}$} & \multicolumn{2}{|c|}{ Época lluviosa } & \multicolumn{2}{|c|}{ Época seca } \\
\hline & & $x$ & $\mathbf{Y}$ & & $\begin{array}{l}\text { Ancho } \\
\text { del río } \\
(\mathrm{m})\end{array}$ & $\begin{array}{l}\text { Profundidad } \\
(\mathrm{m})\end{array}$ & \begin{tabular}{l}
\multicolumn{2}{l}{ Ancho } \\
del río \\
$(\mathrm{m})$
\end{tabular} & $\begin{array}{l}\text { Profundidad } \\
\text { (m) }\end{array}$ \\
\hline P1 & $\begin{array}{l}\text { Río moderadamente } \\
\text { torrentoso, aguas } \\
\text { transparentes. Lecho con } \\
\text { grava y piedras; árboles de } \\
20 \text { m de alto, arbustos y } \\
\text { helechos. }\end{array}$ & $\begin{array}{l}17 \mathrm{M} \\
0737693\end{array}$ & 20505 & 1050 & 8,5 & 0,7 & 5,5 & 0,7 \\
\hline P2 & $\begin{array}{l}\text { Río torrentoso, con lecho } \\
\text { de piedras. Las orillas tienen } \\
\text { vegetación que cubren el } \\
\text { lecho del río, como árboles } \\
\text { de } 30 \mathrm{~m} \text { de alto, arbustos, } \\
\text { helechos y plantas } \\
\text { herbáceas. }\end{array}$ & $\begin{array}{l}17 \mathrm{M} \\
0737649\end{array}$ & 20712 & 934 & 9,5 & 0,6 & 6,5 & 0,63 \\
\hline P3 & $\begin{array}{l}\text { Río torrentoso, con lecho de } \\
\text { piedras. Las orillas tienen } \\
\text { vegetación secundaria, } \\
\text { compuesta por pequeños } \\
\text { arbustos y árboles } \\
\text { pequeños }(10 \mathrm{~m}) \text { con pocas } \\
\text { epifitas }\end{array}$ & $\begin{array}{l}17 \mathrm{M} \\
0737712\end{array}$ & 20867 & 928 & 7,3 & 0,5 & 5,3 & 0,5 \\
\hline P4 & $\begin{array}{l}\text { Río moderadamente } \\
\text { torrentoso, lecho con } \\
\text { piedras y grava. Las orillas } \\
\text { tienen pocos árboles y } \\
\text { arbustos. Presencia de } \\
\text { pasto y residuos sólidos }\end{array}$ & $\begin{array}{l}17 \mathrm{M} \\
0737490\end{array}$ & 20270 & 900 & 8,5 & 0,6 & 5,2 & 0,6 \\
\hline P5 & $\begin{array}{l}\text { Río moderadamente } \\
\text { torrentoso, lecho con } \\
\text { piedras y grava. Las orillas } \\
\text { tienen vegetación } \\
\text { secundaria, pocos árboles y } \\
\text { arbustos. Uso del río como } \\
\text { cruce de vía secundaria } \\
\text { para vehículos motorizados. }\end{array}$ & $\begin{array}{l}17 \mathrm{M} \\
0737843\end{array}$ & 21008 & 893 & 10,0 & 0,2 & 7,3 & 0,2 \\
\hline
\end{tabular}

\section{Técnica Manual}

Consiste en encontrar organismos adheridos al sustrato, para esto se levantaron y analizaron piedras, rocas, ramas sumergidas y hojarasca de la ribera del río, en un período de tiempo de 10 minutos (Nugra et. al., 2016). Los macroinvertebrados fueron recolectados mediante una pinza entomológica y depositados directamente en viales plásticos de $10 \mathrm{ml}$ con contenido de alcohol al $70 \%$, previamente etiquetados con la fecha, lugar y número de punto de muestreo, para su posterior identificación. Esta técnica se llevó a cabo una vez por cada punto seleccionado.

\section{Técnica de patada}

Utiliza una red de patada, ojo de malla de 0,5 a $1 \mathrm{~mm}$. Consiste en colocar los dos extremos de la red en el fondo del río a contra corriente y atrapar los macroinvertebrados en la red al remover el sustrato con los pies, durante un tiempo determinado de 5 minutos aproximadamente (Carrera et. al., 2001). Esta técnica se llevó a cabo dos veces por cada punto seleccionado. 


\section{Técnica Surber}

Emplea una red Surber, ojo de malla de 0,5 a $1 \mathrm{~mm}$. Consiste en colocar la red en el fondo del río a contra corriente, mientras se remueve el sustrato del fondo con las manos, durante un minuto (Carrera et. al., 2001). La red "consta de un marco metálico de 30 x $30 \mathrm{~cm}$, al cual está sujeta una red de unos $80 \mathrm{~cm}$ de longitud y con una abertura de malla de aproximadamente $500 \mu$ (Universidad Nacional Mayor de San Marcos [UNMSM], 2014 págs. 37 - 42). Esta técnica se llevó a cabo dos veces por cada punto seleccionado.

Las muestras colectadas en los cinco puntos de muestreo, con las técnicas de red de patada y red Surber, fueron colocadas en bandejas de loza blanca con la finalidad de separar los organismos del material más grande; a continuación se colocaron las muestras en fundas herméticas previamente etiquetadas y finalmente se puso en hieleras para su posterior traslado e identificación.

Para la recolección de datos de los parámetros físico - químicos, se empleó el equipo multiparámetro portátil $\mathrm{HACH}$, que permitió la medición de temperatura $\left({ }^{\circ} \mathrm{C}\right), \mathrm{pH}$, conductividad $\left(\mathrm{uS} / \mathrm{cm}^{2}\right)$ y oxígeno disuelto $(\mathrm{mg} / \mathrm{L})$; además se utilizó el turbidímetro que midió la turbidez (NTU) del agua.

Para el análisis de $\mathrm{DBO}_{5}\left(\mathrm{mgO}_{2} / \mathrm{L}\right)$ y $\mathrm{DQO}\left(\mathrm{mgO}_{2} / \mathrm{L}\right)$, se tomaron muestras de agua, empleando 5 botellas de vidrio de $100 \mathrm{ml}$, previamente esterilizadas y etiquetadas con fecha y código de muestra. Una vez las muestras fueron recolectadas, se procedió a cerrarlas de manera hermética. Luego se almacenaron en hieleras previamente refrigeradas para su posterior traslado.

Tanto la recolección de datos in-situ como la toma de muestras de agua fueron llevadas a cabo en cada punto de muestreo, en las dos épocas climáticas.

Adicionalmente en cada punto de muestreo se tomaron datos de la profundidad y ancho del cuerpo de agua tanto en época lluviosa como en seca.

\subsection{Fase de laboratorio}

Las muestras preservadas en alcohol, procedentes de los cinco puntos de muestreo, en cada época climática fueron colocadas en cajas Petri para proceder a la observación a través del estereomicroscopio con aumentos de $0.7 x$ y $4.5 x$. Se empleó una pinza entomológica y una aguja de disección para la identificación de los especímenes según su orden y familia, utilizando las claves taxonómicas de identificación como Roldán 1988; Carrera \& Fierro, 2001; Domínguez \& Fernández, 2009; Nugra et. al., 2016.

Los análisis $\mathrm{DBO}_{5}$ y el $\mathrm{DQO}$, fueron realizados en el laboratorio OSP de la Universidad Central del Ecuador (UCE), a partir de los siguientes métodos certificados: DBO5: MAM - 38 / APHA 5210 B Modificado y DQO: MAM - 23 A / MERK 112, 28, 29, 132 Modificado.

Se compararon los parámetros obtenidos tanto in-situ como en los laboratorios con los parámetros considerados en la normativa ambiental vigente: TULSMA, libro VI. Adicionalmente, y bajo la misma normativa, se determinó si los límites de descarga a un cuerpo de agua dulce se encuentran dentro de los límites máximos permisibles (Texto Unificado de Legislación Secundaria del Medio Ambiente [TULSMA], 2015).

\subsection{Análisis de datos}

Se realizaron los siguientes cálculos: Abundancia Total $(\mathrm{N})$ : número total de individuos colectados en cada punto de muestreo (Álvarez et al., 2006); Abundancia (n): número de individuos por cada taxón colectado; Abundancia Relativa (\%): número de individuos de cada taxón dividido para la abundancia total $(N)$ por cien (Guiracocha, 2000); Riqueza (S): número total de taxones registrados en cada punto de muestreo (Bode, 1988); Indice BMWP/Col, da el valor de 1 a 10 a cada familia de 
macroinvertebrados de acuerdo con su tolerancia a la contaminación (Roldán, 2003); Índice EPT, considera a los grupos de Ephemeroptera, Plecoptera, Trichoptera, indicadores de la calidad del agua debido a su sensibilidad a los contaminantes (Carrera et. al., 2001).

Para el análisis de los componentes principales (PCA en Inglés) se utilizó el programa SIMCA versión 15 University License (UT-S-15-05).

\section{Resultados}

\subsection{Ancho y profundidad del Río Sardinas}

Se registró el ancho y la profundidad del río en cada uno de los cinco puntos de muestreo en las épocas seca y lluviosa. Respecto al ancho de los puntos de muestreo, no se observaron diferencias durante época seca y época lluviosa; mientras que, la profundidad si mostró diferencias en los valores obtenidos en ambas estaciones, evidenciando que el cuerpo de agua estudiado es menos profundo en época seca (Tabla 1).

\subsection{Análisis físico - químico}

En la Tabla 2 se encuentra representada la comparación entre los parámetros físico-químicos y los límites máximos permisibles (TULSMA, 2015).

Tabla 2. Comparación de los Parámetros Físico - Químicos, época lluviosa y seca

\begin{tabular}{|c|c|c|c|c|c|c|c|c|c|c|c|c|c|c|c|c|}
\hline \multicolumn{2}{|l|}{ Medición } & \multicolumn{7}{|c|}{ Época lluviosa } & \multirow{2}{*}{\begin{tabular}{|l|}
$\begin{array}{l}\text { Límite } \\
\text { Máx. } \\
\text { permisible }\end{array}$ \\
(TULSMA)
\end{tabular}} & \multicolumn{7}{|c|}{ Época seca } \\
\hline Parámetro & Unidad & P1 & $\mathrm{P} 2$ & P3 & P4 & P5 & $\bar{x}$ & $\sigma$ & & $\mathrm{PI}$ & P2 & P3 & P4 & P5 & $\overline{\mathrm{x}}$ & $\sigma$ \\
\hline Temperatura & $\stackrel{\circ}{\circ}$ & 22,8 & 23,1 & 23,3 & 23,2 & 23,4 & 23,2 & 0,2 & Máx. 32 & 23,7 & 24,1 & 23,8 & 24 & 24,3 & 24,0 & 0,2 \\
\hline Conductividad & $\mu \mathrm{S} / \mathrm{cm}^{2}$ & 4,4 & 4,4 & 4,5 & 4,5 & 4,5 & 4,4 & 0,1 & $100-2000$ & 21,9 & 22,0 & 22,0 & 21,9 & 22,0 & 22,0 & 0,1 \\
\hline Turbidez & NTU & 2,1 & 3,2 & 3,4 & 2,0 & 3,9 & 2,9 & 0,8 & $0-50$ & 1,2 & 1,9 & 1,5 & 2,0 & 3,6 & 2,0 & 0,9 \\
\hline O.D. & $\mathrm{mg} / \mathrm{L}$ & 7,3 & 7,1 & 6,9 & 7,0 & 6,0 & 6,8 & 0,5 & $>6$ & 6,7 & 6,6 & 6,7 & 6,6 & 6,0 & 6,5 & 0,3 \\
\hline $\mathrm{pH}$ & & 6,0 & 6,5 & 6,0 & 6,7 & 6,0 & 6,2 & 0,3 & 06-sep & 6,3 & 6,0 & 6,0 & 6,0 & 6,0 & 6,1 & 0,1 \\
\hline DQO & $\mathrm{mgO}_{2} / \mathrm{L}$ & & & & & & $<8$ & & 250 & & & & & & $<8$ & \\
\hline $\mathrm{DBO}_{5}$ & $\mathrm{mgO}_{2} / \mathrm{L}$ & & & & & & $<5$ & & & & & & & & $<5$ & \\
\hline
\end{tabular}

\section{Temperatura}

El límite máximo permisible para temperatura del agua es de $32^{\circ} \mathrm{C}$. El río cumple con estas especificaciones tanto en la época lluviosa $\left(23.4^{\circ} \mathrm{C}\right)$ como en la seca $\left(24.0^{\circ} \mathrm{C}\right)$; el aumento de temperatura se encuentra ligada a la influencia de las condiciones climáticas del área.

\section{Conductividad}

Los límites máximos permisibles van de 100 - $2000 \mathrm{uS} / \mathrm{cm}^{2}$, que se cumple tanto en la época lluviosa (4.4) como en la seca (22.0). En ambas épocas la conductividad va en aumento a partir del P1 (escasos sólidos disueltos); sin embargo, se estabiliza en los puntos restantes. El agua dulce que se pierde por evaporación aumenta la conductividad y la salinidad de la masa de agua porque la cantidad de iones disueltos aumentan los valores de ambas (Water Boards, 2018). 


\section{Turbidez}

Los límites máximos permisibles van de 0 a 50 NTU, cumple tanto en la época lluviosa (2.9) como en la seca (2.0). La turbidez disminuye en la época seca debido a la disminución del caudal en comparación de la lluviosa que posee mayor presencia de sedimentos, sustratos y descomposición de hojarasca.

\section{Oxígeno Disuelto (O.D.)}

El límite máximo permisible es de $>6 \mathrm{mg} / \mathrm{L}$; cumple tanto en la época lluviosa (6.8) como en el seca (6.5). El O.D., posee relación con la temperatura de agua y disminuye con ella; se deduce que el agua es apta para desarrollar vida (TULSMA, 2015).

pH

El límite máximo permisible va de 6 - 9; cumple tanto en la época lluviosa (6.2) como en la seca (6.1). En las dos épocas climáticas se obtuvo un pH ácido categorizándolo como un nivel normal en este río.

\section{Demanda química de oxígeno (DQO)}

El límite máximo permisible es de $250 \mathrm{mgO}_{2} / \mathrm{L}$; cumple en la época lluviosa $(<8)$ y en la seca $(<8)$. Sin embargo, los valores de las dos épocas climáticas determinaron que el agua presenta una contaminación leve, este criterio se debe a que las aguas no contaminadas de ríos suelen tener un valor de 1 a $5 \mathrm{mgO}_{2} / \mathrm{L}$ (Restrepo et. al., 2002).

\section{Demanda bioquímica de oxígeno $\left(\mathrm{DBO}_{5}\right)$}

El límite máximo permisible es de $100 \mathrm{mgO}_{2} / \mathrm{L}$; cumple en la en la época lluviosa $(<5)$ y en la seca $(<5)$. Estas cifras reflejan la existencia de fauna acuática de gran riqueza y diversidad en los cinco puntos de muestreo (Empresa Pública Municipal de Telecomunicaciones, Agua Potable, Alcantarillado y Saneamiento [ETAPA], 2008).

\subsection{Abundancia y Riqueza de macroinvertebrados}

Se recolectaron un total de 526 individuos, 265 en época lluviosa y 261 en época seca que corresponden a 5 Clases, 13 órdenes y 38 familias, 31 en la estación lluviosa y 30 en la estación seca. En época lluviosa el taxón más abundante fue Leptophlebiidae con un total de 34 individuos que corresponde al 12,8\% de los especímenes colectados. En la estación seca el taxón más abundante fue Chironomidae con 45 individuos que corresponde al 17,2\% de los individuos colectados (Tabla 3).

En época lluviosa el punto de muestreo P1 fue el más abundante con 72 individuos colectados. El taxón más numeroso de este punto fue Elmidae con 12 individuos que corresponden al $16,7 \%$ de los individuos capturados y los menos abundantes fueron Chironomidae, Perlidae, Hydropsychidae y Sericostomatidae con tres individuos cada taxón (4,2\%). El punto P5 fue el menos cuantioso con 37 especímenes colectados; el taxón más representativo fue Lepthophlebiidae con $14 \quad(37,8 \%)$ individuos y los menos representativos: Tubificidae, Chironomidae, Corydalidae, Aeshnidae, Coenagrionidae, Perlidae y Planariidae con un espécimen colectado $(2,7 \%)$. En cuanto a la riqueza el punto $\mathrm{P} 2$ tuvo el mayor número de taxones 12 y el menos diverso fue el punto 4 con 12 taxones. 
Tabla 3. Riqueza y abundancia de macroinvertebrados acuáticos de los puntos de muestreo en el Río Sardinas, durante época lluviosa y época seca

\begin{tabular}{|c|c|c|c|c|c|c|c|c|c|c|c|c|c|c|c|c|}
\hline \multirow[b]{2}{*}{ Clase } & \multirow[b]{2}{*}{ Orden } & \multirow[b]{2}{*}{ Familia } & \multicolumn{7}{|c|}{ Época lluviosa } & \multicolumn{7}{|c|}{ Época seca } \\
\hline & & & P1 & P2 & P3 & P4 & P5 & Total & $\%$ & P1 & P2 & P3 & P4 & P5 & Total & $\%$ \\
\hline \multirow[b]{2}{*}{ Oligochaeta } & \multirow[b]{2}{*}{ Haplotaxida } & Tubificidae & 0 & 2 & 0 & 0 & 1 & 3 & 1,1 & 0 & 0 & 0 & 0 & 0 & 0 & 0,0 \\
\hline & & Naididae & 0 & 1 & 0 & 1 & 0 & 2 & 0,8 & 0 & 0 & 0 & 0 & 0 & 0 & 0,0 \\
\hline \multirow[b]{3}{*}{ Malacostraca } & \multirow[b]{2}{*}{ Isopoda } & Asellidae & 0 & 0 & 0 & 0 & 0 & 0 & 0,0 & 1 & 0 & 0 & 0 & 0 & 1 & 0,4 \\
\hline & & Cymothoidae & 0 & 0 & 0 & 0 & 0 & 0 & 0,0 & 0 & 1 & 0 & 0 & 0 & 1 & 0,4 \\
\hline & Amphipoda & Hyalellidae & 0 & 0 & 0 & 0 & 0 & 0 & 0,0 & 2 & 0 & 0 & 0 & 0 & 2 & 0,8 \\
\hline \multirow[t]{31}{*}{ Arachnoidea } & Hydracarina & Hydrachnidae & 0 & 0 & 0 & 0 & 0 & 0 & 0,0 & 2 & 0 & 0 & 0 & 0 & 2 & 0,8 \\
\hline & \multirow[b]{6}{*}{ Coleóptera } & Dryopidae & 0 & 0 & 1 & 0 & 0 & 1 & 0,4 & 0 & 0 & 0 & 0 & 0 & 0 & 0,0 \\
\hline & & Elmidae & 12 & 3 & 0 & 0 & 2 & 17 & 6,4 & 14 & 10 & 9 & 3 & 2 & 38 & 14,6 \\
\hline & & Hydrophilidae & 0 & 0 & 0 & 4 & 0 & 4 & 1,5 & 3 & 0 & 0 & 2 & 0 & 5 & 1,9 \\
\hline & & Psephenidae & 0 & 3 & 0 & 0 & 0 & 3 & 1,1 & 0 & 0 & 0 & 1 & 2 & 3 & 1,1 \\
\hline & & Limnichidae & 0 & 0 & 0 & 0 & 0 & 0 & 0,0 & 0 & 1 & 0 & 0 & 0 & 1 & 0,4 \\
\hline & & Staphylinidae & 0 & 0 & 0 & 0 & 0 & 0 & 0,0 & 1 & 0 & 0 & 0 & 0 & 1 & 0,4 \\
\hline & \multirow[b]{4}{*}{ Díptera } & Ceratopogonidae & 0 & 4 & 10 & 0 & 0 & 14 & 5,3 & 3 & 3 & 0 & 0 & 0 & 6 & 2,3 \\
\hline & & Culicidae & 0 & 0 & 1 & 0 & 0 & 1 & 0,4 & 2 & 3 & 8 & 9 & 4 & 26 & 10,0 \\
\hline & & Chironomidae & 3 & 12 & 8 & 0 & 1 & 24 & 9,1 & 4 & 12 & 14 & 10 & 5 & 45 & 17,2 \\
\hline & & Tipulidae & 0 & 0 & 6 & 6 & 6 & 18 & 6,8 & 0 & 10 & 2 & 0 & 0 & 12 & 4,6 \\
\hline & \multirow[b]{4}{*}{ Ephemeróptera } & Baetidae & 0 & 1 & 0 & 4 & 2 & 7 & 2,6 & 5 & 4 & 0 & 6 & 7 & 22 & 8,4 \\
\hline & & Caenidae & 0 & 3 & 0 & 2 & 0 & 5 & 1,9 & 0 & 0 & 0 & 0 & 0 & 0 & 0,0 \\
\hline & & Leptophlebiidae & 8 & 5 & 7 & 0 & 14 & 34 & 12,8 & 8 & 4 & 2 & 2 & 8 & 24 & 9,2 \\
\hline & & Leptohyphidae & 8 & 0 & 0 & 0 & 0 & 8 & 3,0 & 0 & 2 & 1 & 0 & 0 & 3 & 1,1 \\
\hline & \multirow[b]{2}{*}{ Hemíptera } & Naucoridae & 0 & 0 & 0 & 0 & 0 & 0 & 0,0 & 3 & 0 & 0 & 0 & 0 & 3 & 1,1 \\
\hline & & Veliidae & 4 & 0 & 0 & 7 & 2 & 13 & 4,9 & 0 & 0 & 0 & 2 & 0 & 2 & 0,8 \\
\hline & Megalóptera & Corydalidae & 0 & 0 & 0 & 0 & 1 & 1 & 0,4 & 0 & 0 & 0 & 0 & 0 & 0 & 0,0 \\
\hline & \multirow[b]{4}{*}{ Odonata } & Aeshnidae & 0 & 0 & 0 & 0 & 1 & 1 & 0,4 & 0 & 0 & 0 & 0 & 0 & 0 & 0,0 \\
\hline & & Coenagrionidae & 0 & 0 & 0 & 3 & 1 & 4 & 1,5 & 0 & 1 & 0 & 1 & 0 & 2 & 0,8 \\
\hline & & Gomphidae & 0 & 0 & 2 & 0 & 0 & 2 & 0,8 & 0 & 1 & 1 & 0 & 0 & 2 & 0,8 \\
\hline & & Libellulidae & 5 & 0 & 1 & 0 & 2 & 8 & 3,0 & 5 & 0 & 0 & 0 & 2 & 7 & 2,7 \\
\hline & Plecóptero & Perlidae & 3 & 0 & 0 & 0 & 1 & 4 & 1,5 & 0 & 0 & 1 & 2 & 0 & 3 & 1,1 \\
\hline & \multirow{9}{*}{ Trichóptera } & Glossosomatidae & 4 & 4 & 0 & 0 & 0 & 8 & 3,0 & 0 & 0 & 0 & 0 & 2 & 2 & 0,8 \\
\hline & & Hydrobiosidae & 0 & 0 & 1 & 0 & 0 & 1 & 0,4 & 0 & 0 & 0 & 0 & 0 & 0 & 0,0 \\
\hline & & Hydropsychidae & 3 & 2 & 1 & 2 & 0 & 8 & 3,0 & 3 & 3 & 2 & 0 & 3 & 11 & 4,2 \\
\hline & & Leptoceridae & 9 & 12 & 5 & 3 & 2 & 31 & 11,7 & 5 & 1 & 4 & 4 & 0 & 14 & 5,4 \\
\hline & & Limnephilidae & 2 & 1 & 3 & 4 & 0 & 10 & 3,8 & 0 & 0 & 0 & 0 & 1 & 1 & 0,4 \\
\hline & & Odontoceridae & 4 & 4 & 6 & 0 & 0 & 14 & 5,3 & 0 & 0 & 0 & 0 & 0 & 0 & 0,0 \\
\hline & & Polycentropodidae & 4 & 0 & 4 & 1 & 0 & 9 & 3,4 & 2 & 2 & 7 & 0 & 0 & 11 & 4,2 \\
\hline & & Philopotamidae & 0 & 0 & 0 & 3 & 0 & 3 & 1,1 & 2 & 2 & 2 & 0 & 0 & 6 & 2,3 \\
\hline Insecta & & Sericostomatidae & 3 & 3 & 0 & 0 & 0 & 6 & 2,3 & 3 & 0 & 0 & 0 & 0 & 3 & 1,1 \\
\hline \multirow[t]{3}{*}{ Turbellaria } & Tricladida & Planariidae & 0 & 0 & 0 & 0 & 1 & 1 & 0,4 & 0 & 0 & 0 & 0 & 2 & 2 & 0,8 \\
\hline & & Abundancia $(\mathrm{N})$ & 72 & 60 & 56 & 40 & 37 & 265 & 100 & 68 & 60 & 53 & 42 & 38 & 261 & 100,0 \\
\hline & & Riqueza (S) & 14 & 15 & 14 & 12 & 14 & 38 & & 18 & 16 & 12 & 11 & 11 & 38 & \\
\hline
\end{tabular}


En la época seca el punto P1 fue el más abundante con 68 individuos colectados. La familia más representativa fue Elmidae con 14 individuos capturados correspondiente al $21,5 \%$ de la muestra colectada. La menos numerosa fue Staphylinidae con un individuo, que corresponde al 1,5\%. El punto P5 fue el menos cuantioso con 38 especímenes colectados. Leptophlebiidae fue el taxón más abundante $21,1 \%$ y el menos numeroso Limnephilidae con un individuo (2,6\%). El punto 1 tuvo el mayor número de taxones 18 y el menos diverso fue P4 y P5 con 11 individuos cada uno.

\section{4. Índice BMWP/Col}

En la época lluviosa los puntos P1 y P2 obtuvieron valores altos del Índice BMWP/Col (Roldán, 2003), es decir que su calidad de agua es buena (aguas limpias), gracias a la presencia y abundancia de taxones de los órdenes Ephemeroptera y Trichoptera. Mientras que, los puntos P3, P4 y P5, obtuvieron valores moderados, es decir, que tienen una calidad de agua aceptable (ligeramente contaminada). Se evidencia la disminución de familias, en especial de los órdenes Ephemeroptera y Trichoptera, así como se acentúa la presencia de macroinvertebrados más tolerantes a la contaminación, como aquellos de los órdenes Coleoptera y Diptera. En la estación seca el P1 tuvo calidad buena y los puntos P2 - P5, se encuentran en un rango de calidad de agua aceptable (Tabla 4).

Tabla 4. Valores del Índice BMWP/Col obtenidos en los puntos de muestreo del río Sardinas, en época lluviosa y época seca.

\begin{tabular}{|c|c|c|c|c|c|c|}
\hline \multicolumn{4}{|c|}{ Época lluviosa } & \multicolumn{3}{|l|}{ Época seca } \\
\hline $\begin{array}{l}\text { Puntos de } \\
\text { muestreo }\end{array}$ & $\begin{array}{l}\text { Índice } \\
\text { BMWP/Col }\end{array}$ & $\begin{array}{l}\text { Calidad del } \\
\text { Agua }\end{array}$ & Significado & $\begin{array}{l}\text { Índice } \\
\text { BMWP/Col }\end{array}$ & $\begin{array}{l}\text { Calidad del } \\
\text { Agua }\end{array}$ & Significado \\
\hline P1 & 118 & & Aguas limpias & 103 & Buena & Aguas limpias \\
\hline P2 & 104 & Buena & Aguas limpias & 91 & \multirow{4}{*}{ Aceptable } & $\begin{array}{l}\text { Aguas ligeramente } \\
\text { contaminadas }\end{array}$ \\
\hline P3 & 94 & \multirow{3}{*}{ Aceptable } & $\begin{array}{l}\text { Aguas } \\
\text { ligeramente } \\
\text { contaminadas }\end{array}$ & 85 & & $\begin{array}{l}\text { Aguas ligeramente } \\
\text { contaminadas }\end{array}$ \\
\hline P4 & 88 & & $\begin{array}{l}\text { Aguas } \\
\text { ligeramente } \\
\text { contaminadas }\end{array}$ & 78 & & $\begin{array}{l}\text { Aguas ligeramente } \\
\text { contaminadas }\end{array}$ \\
\hline P5 & 84 & & $\begin{array}{l}\text { Aguas } \\
\text { ligeramente } \\
\text { contaminadas }\end{array}$ & 69 & & $\begin{array}{l}\text { Aguas ligeramente } \\
\text { contaminadas }\end{array}$ \\
\hline
\end{tabular}

Al analizar los muestreos realizados en las dos épocas climáticas (Figura 2), se observó que el punto 1, mantiene la calidad de agua buena, tanto en época lluviosa como en época seca, a pesar que los valores decayeron. El punto 2, disminuyó su calidad de agua en época seca, pasando de buena a aceptable. Los puntos 3,4 y 5 mantuvieron su estatus de calidad de agua aceptable durante las dos épocas de muestreo. El caudal hidrológico del río Sardinas varió durante las dos épocas, lo cual se evidenció en los datos de profundidad registrados. Esta modificación ecológica influye directamente en la disposición de microhábitats y fuentes de alimentos para los macroinvertebrados, por lo tanto, su diversidad, composición y presencia de organismos sensibles y tolerantes se ve afectada, influyendo en la calidad del agua que reflejan (Ríos-Touma et. al., 2011). 


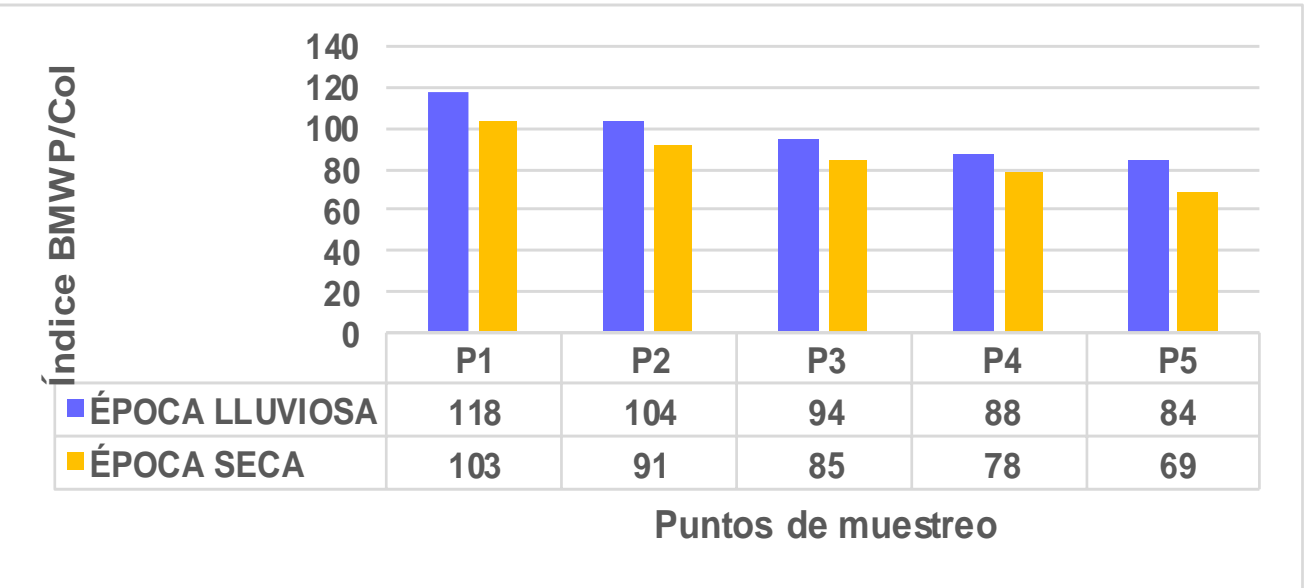

Figura 2. Comparación de los valores del Índice BMWP/Col de los puntos de muestreo en el río Sardinas, épocas lluviosa y seca

\section{5. Índice Ephemeroptera, Trichoptera y Plecoptera (EPT)}

En la época lluviosa se obtuvieron un total de 148 especímenes EPT y 100 en la época seca, lo cual da una abundancia relativa de $56 \%$ en lluvias, categorizándola como buena y $38 \%$ en la estación seca, categorizándola como regular (Carrera et. al., 2001).

En la Figura 3, se puede apreciar la representación gráfica del porcentaje obtenido en el análisis del índice EPT. En la comparación se puede apreciar que la calidad del agua es buena en la época lluviosa, mientras que en la época seca la calidad del agua es regular.

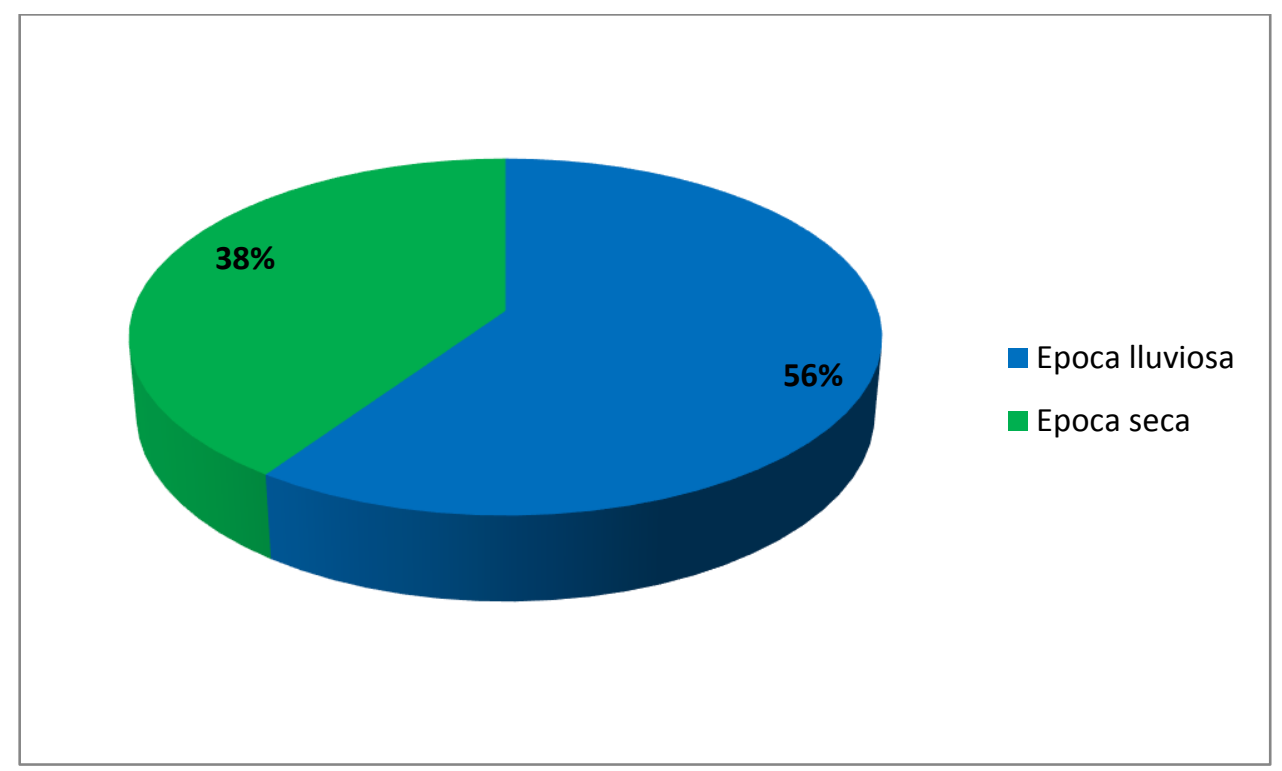

Figura 3. Porcentaje resultante del análisis del índice EPT.

\subsection{Análisis de componentes principales (PCA)}

Se realizó un PCA entre los parámetros físico-químicos sobre los valores de riqueza, abundancia, BMWP/Col y EPT de cada punto de muestreo, durante ambas épocas climáticas; en el cual se pudo observar una mayor abundancia en el punto 1, que corresponde al punto que tiene mayor oxígeno disuelto, menor conductividad y temperatura y el valor más alto de BMWP/Col. Figura 4. 


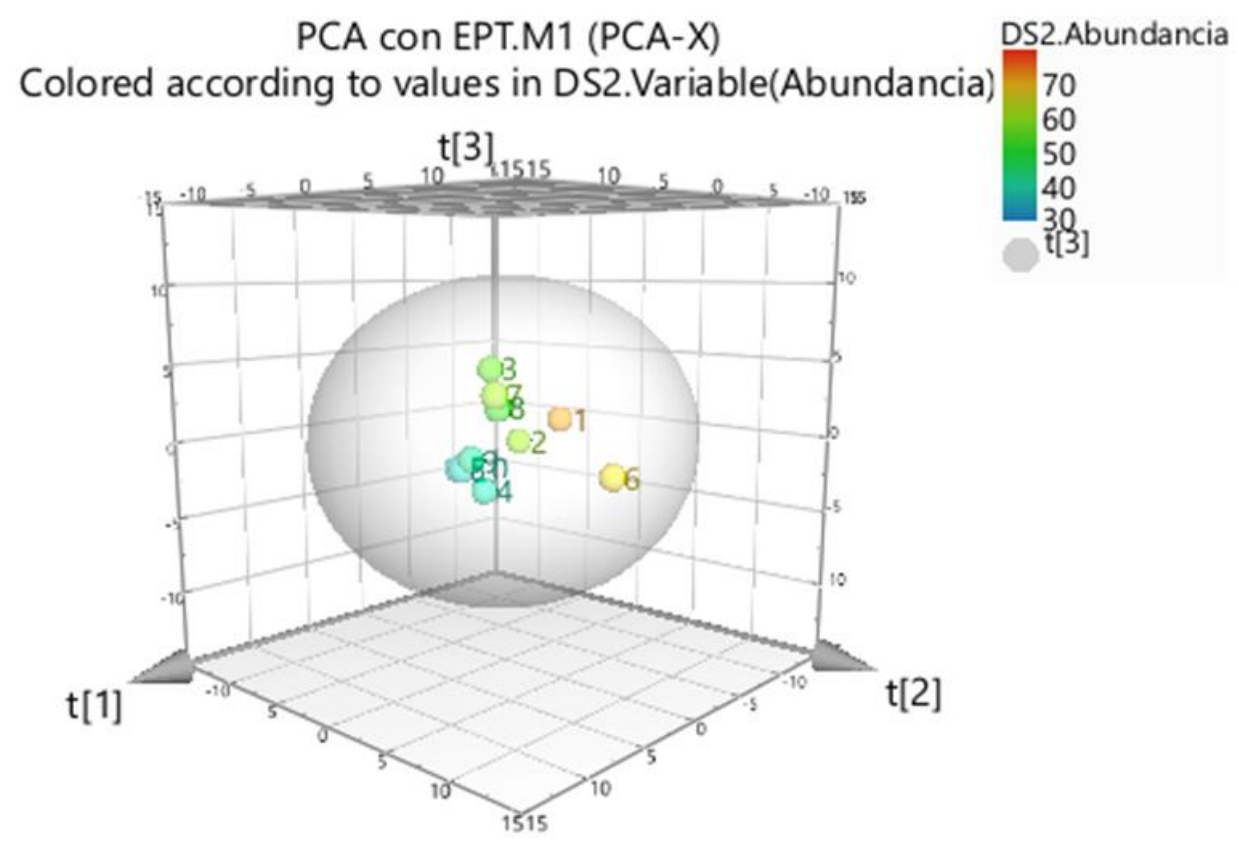

Figura 4. Análisis de Componentes Principales (PCA)

\section{Discusión}

En las dos épocas climáticas se determinó gracias al índice BMWP/Col, que según Arroyo \& Encalada (2009), es el índice ideal para determinar la calidad del agua en el ecosistema montano, debido a que más del $97 \%$ de familias de macroinvertebrados registradas se encuentran incluidas en este índice de calidad. Además de ser el más utilizado para evaluar la integridad de sistemas acuáticos en América Latina (Ramírez \& Gutiérrez-Fonseca, 2014). Que la calidad del agua del río Sardinas es aceptable (aguas ligeramente contaminadas); sin embargo, según el índice ETP la calidad es buena en la época lluviosa, mientras que en la seca la calidad es regular. Esto se debe a la disminución de familias de los tres grupos de macroinvertebrados Ephemeroptera, Trichoptera y Plecoptera.

En una publicación realizada por Ríos-Touma et. al., (2011) en el río Piburja de Ecuador ubicado a $3300 \mathrm{msnm}$ reporta cambios significativos en la composición de la comunidad de macroinvertebrados entre las épocas seca y de lluvias, coincidiendo con los resultados obtenidos en el presente estudio.

De igual modo existe un decrecimiento en la calidad del agua en la época seca lo cual coincide con los estudios de Jacobsen \& Encalada (1998), quienes estudiaron 8 cuerpos de agua alrededor de la ciudad de Quito, Ecuador entre los 2600 y 3100 msnm y Giacometti \& Bersosa (2006) que estudiaron la cuenca del río Alambi entre los 11202610 y msnm., observando, en ambos casos, un decrecimiento del índice de calidad del agua en época seca.

La riqueza (S) para la época lluviosa es 31 y en la seca 30 . Esto determina que no existe mayor cambio en el número de familias colectadas en ambas épocas climáticas. En cuanto a la composición de los macroinvertebrados, sí se hallaron diferencias entre ambas épocas climáticas y en cada punto de muestreo lo cual concuerda con lo establecido por Jacobsen y Encalada (1998).

Los parámetros físico-químicos cumplen con los límites máximos permisibles contemplados con la normativa vigente ambiental TULSMA. No obstante, el DQO $<8$ $\mathrm{mgO} 2 / \mathrm{L}$ de las dos épocas, demostró que aunque dentro de los límites, el río Sardinas se encuentra levemente contaminado al tener un valor superior a $5 \mathrm{mgO}_{2} / \mathrm{L}$ (TULSMA, 
2015). Estos datos coinciden con el trabajo realizado por Endara (2018) en los meses de mayo y junio del 2017.

Mediante el Análisis de Componentes Principales (PCA) se pudo observar que el punto de mayor abundancia es el punto 1 que corresponde al punto con mayor oxígeno disuelto y menor conductividad, lo cual concuerda con lo publicado por Moya et. al. (2009).

\section{Conclusiones y recomendaciones}

Aunque durante el presente estudio la comunidad de macroinvertebrados fue bien caracterizada, es necesario realizar campañas de muestreo interanuales para comprender fluctuaciones naturales causadas por variaciones en el caudal ecológico y por impactos antrópicos que se presentan en este sistema lótico (Jacobsen, 2003; Encalada \& Ríos 2011; Ríos, et. al., 2012). Las condiciones ecológicas del río Sardinas son, en general, moderadas, con la existencia y la presencia de grupos sensibles como los Trichopteros y Ephemeropteros; sin embargo, la presencia de grupos resistentes a la contaminación como los Chironomidae que son abundantes en el punto P2 en ambas épocas del año y punto P3 en época seca hace que la evaluación de la calidad del agua del río Sardinas sea moderada.

\section{Bibliografía}

Abarca H. (2007). El uso de macroinvertebrados como bioindicadores de la calidad de agua, Biocenosis, 20(1-2).

Álvarez, M., Córdoba, S., Escobar, F., Fagua, G., Gast, F., Mendoza, H., Ospina M., Umaña Ana María, Villarreal, H. (2006). Manual de métodos para el desarrollo de inventarios de Biodiversidad. Bogotá: Recursos Biológicos Alexander Von Humbolt.

Arroyo, C., \& Encalada, A. C. (2009). Evaluación de la calidad de agua a través de macroinvertebrados bentónicos e índices biológicos en ríos tropicales en bosque de neblina montano, ACl Avances en Ciencias e Ingenierías, 1(1).

Carrera, C., \& Fierro, K. (2001). Manual de monitoreo: los macroinvertebrados acuáticos como indicadores de la calidad del agua. Quito: EcoCiencia.

Domínguez, E., \& Fernández, H. (2009). Macroinvertebrados bentónicos sudamericanos: sistemática y biología. Tucumán, Argentina: Fundación Miguel Lillo.

Empresa Pública Municipal de Telecomunicaciones, Agua Potable, Alcantarillado y Saneamiento [ETAPA]. (2008). Evolución de la calidad del agua de los tramos bajos de los ríos de la ciudad de Cuenca 2008. Cuenca: Dirección de Gestión Ambiental. Recuperado de http://www.etapa.net.ec/Portals/0/LeyTransparencia/Auditoria/ InformeUAI-0061-2012.pdf

Encalada, A \& Ríos, B. (2011). Aprendizaje de las cuencas altoandinas: Proyecto FUCARA, Laboratorio de Ecología Acuática - USFQ, Quito.

Endara A. 2018. Evaluation of Water Quality through benthic macroinvertebrates in the Sardinas River and La Delicia stream in Ecuador. Bulletin of Science and Practice 4 (8), 10-17.

Giacometti, J., \& Bersosa, F. (2006). Macroinvertebrados acuáticos y su importancia como bioindicadores de calidad del agua en el río Alambi, Boletín Técnico, 6, 17-32.

Guiracocha, G. (2000). Conservación de la biodiversidad en los sistemas agroforestales cacaoteros y bananeros de Talamanca, Costa Rica. Turrialba. Recuperado de https://books.google.com.ec/books?id=6n8zAQAAMAAJ\&pg=PA19\&lpg=PA19\&dq= $\% 25 \% 3 \mathrm{DNi} / \mathrm{N}+\mathrm{x} 100+\% 25 \% 3 \mathrm{DNi} / \mathrm{N}+\times 100+$ abundancia+relativa\&source=bl\&ots=QiQ ALQBstF\&sig=Y5koHXN5Pc60A8Jt9uq0s65FtrM\&hl=es419\&sa $=X \& v e d=2 a h U K E w j r 2 Y r E h 4 f d A h X I t \mid k K H Y \_R B B c Q 6 A E w A n o E C A g Q A Q \# v=0$ nepage 
166

Herrera A., (2018). Estudio de prefactibilidad de una finca agroecoturística en la comunidad La Delicia, parroquia de Pacto, cantón Quito. Trabajo de titulación previo a la obtención del Título de Licenciada en Turismo Ecológico. Carrera de Turismo Ecológico. Quito, Universidad Central del Ecuador.

Jacobsen D., y Encalada A., (1998). The macroinvertebrate fauna of Ecuadorian Highland streams in the wet and dry seasons, Hidrobiologie 142 (1) 53-70pp.

Jacobsen, D. (2003). Altitudinal changes in diversity of macroinvertebrates from small streams in the Ecuadorian Andes, Archiv für Hydrobiologie, 158(2), 145-167.

Ministerio de Agricultura y Ganadería - Instituto Espacial Ecuatoriano [MAGAP - IEE]. (2013). Memoria Técnica Cantón Quito Proyecto: "GENERACIÓN DE GEOINFORMACIÓN PARA LA GESTIÓN DEL TERRITORIO A NIVEL NACIONAL ESCALA 1:25.000". Quito. Recuperado de app.sni.gob.ec/snilink/sni/PDOT/...DEL.../IEE/.../mt_quito_clima_hidrologia.doc

Ministerio del Ambiente del Ecuador. 2013. Sistema de Clasificación de los Ecosistemas del Ecuador Continental. Subsecretaría de Patrimonio Natural. Quito.

Moreno, C. E. (2001). ORCYT-UNESCO Oficina Regional de Ciencia y Tecnología para América Latina y el Caribe, UNESCO. Sociedad Entomológica Aragonesa (SEA).

Moya N., Gibon F., Oberdorff T., Rosales C., Domínguez E., (2009). Comparación de las comunidades de macroinvertebrados acuáticos en ríos intermitentes y permanentes del altiplano Boliviano: Implicaciones para el futuro cambio climático. Ecología Aplicada, 8(2), 105 - 114.

Nugra, F., Segovia, E., Benítez, M., \& Reinoso, D. (2016). Guía metodológica para el biomonitoreo de macroinvertebrados e ictiofauna en la Cuenca del Río Napo, Ecuador. Cuenca, ECUADOR: SENAGUA, OTCA.

Plan de Desarrollo y Ordenamiento Territorial de la Parroquia de Pacto [PDOT Pacto]. (2015 - 2019). Junta Parroquial de Pacto: Diagnóstico de la Parroquia de Pacto. Gestnova Cía. Ltda. Recuperado de hhttp://181.112.151.230:8081/attachments /download/656/PDOT\%20PACTO\%202015.pdf

Ramírez, A., \& Gutiérrez-Fonseca, P. E. (2014). Functional feeding groups of aquatic insect families in Latin America: a critical analysis and review of existing literature. Revista de Biología Tropical, 62, 155-167.

Restrepo, A., \& Tobón, L. (2002). Manual de Calidad de Aguas. Escuela de Ingeniería de Antioquia. Medellín, Colombia: Facultad de Ingeniería Ambiental.

Ríos-Touma, B., Encalada, A. C., \& Prat Fornells, N. (2011). Macroinvertebrate Assemblages of an Andean High-Altitude Tropical Stream: The Importance of Season and Flow. International review of hydrobiology, 96(6), 667-685.

Ríos-Touma, B., Prat, N., \& Encalada, A. C. (2012). Invertebrate drift and colonization processes in a tropical Andean stream. Aquatic Biology, 14(3), 233-246.

Roldán, G. (1988). Guía para el estudio de los macroinvertebrados acuáticos del Departamento de Antioquia. Bogotá, Colombia: Pama Editores Ltda.

Roldán, G. (2003). Bioindicación de la calidad del agua en Colombia. Uso del método $B M W P / C o l$ (Primera ed.). Medellín, Colombia: Editorial Universidad de Antioquia.

Texto Unificado de Legislación Secundaria del Medio Ambiente [TULSMA]. (2015). Anexo 1 del Libro VI del TULSMA: Norma de Calidad Ambiental y de Descarga de Efluentes al Recurso Agua. Ministerio del Ambiente Ecuador. Recuperado de https://maeorellana.files.wordpress.com/2015/11/anexo-1-agua.pdf

Universidad Nacional Mayor de San Marcos [UNMSM]. (2014). Métodos de colecta, identificación y análisis de comunidades biológicas: plancton, perifiton, bentos (macroinvertebrados) y necton (peces) en aguas continentales del Perú / Departamento de Limnología, Departamento de Ictiología, Lima: Ministerio del Ambiente.

Villalva, D. (2018). Conferencia para la recolección de datos, río Sardinas. Pacto: "Casa somos Pacto". 
Water Boards. (2018). Folleto Informativo conductividad eléctrica/salinidad. California: Division of Water Quality. Recuperado de https://www.waterboards.ca.gov /water_issues/programs/swamp/docs/.../3130sp.pdf 